\title{
Impediment To Adequate Utilization of Primary Health Care Facilities Among Pregnant Women in Ikpoba-Okha LGA of Edo State
}

\author{
Dr. Enoma Omorogieva IGUDIA DR. (MRS) J.A AGBONIFOH Vero Chinye OMORUYI, \\ Department of Health, Safety and Environmental Education, Faculty of Education, University of Benin, Benin \\ City, Edo State, Nigeria
}

\begin{abstract}
This study was designed to investigate impediments to adequate utilization of Primary Health Care (PHC) facilities by pregnant women in Ikpoba-Okha LGA of Edo State. The descriptive survey design was adopted for the study and a multistage sampling technique was used to select the study sample of 250 respondents. The instruments for data collection were checklist and questionnaire designed by the researchers and built around the research questions. The reliability of the questionnaire was established through test re-test method and a correlation co-efficient of 0.84 was obtained. The researchers led a team of two trained research assistants to administer the instrument to the women during antenatal care services in their PHC Centres. The instrument was then retrieved immediately after completion. Data were analysed using frequency counts and simple percentages. The results of the study showed that: socio-cultural issues, attitudes of health staff, infrastructural decay, accessibility and traditional beliefs affect adequate utilization of PHC facilities by pregnant women in IkpobaOkha. Based on the findings of the study, it is recommended that health education be intensified in creating public awareness on the importance of using PHC facilities among pregnant women and that harmful traditions should be discarded.
\end{abstract}

Keywords: Impediment, Adequate Utilization, PHC, Pregnant Women

DOI: $10.7176 / \mathrm{JEP} / 10-17-13$

Publication date:June $30^{\text {th }} 2019$

\section{INTRODUCTION}

The term Primary Health Care (PHC) is a strategy adopted by the World Health Organization (WHO, 1978) in Russia at the Alma Ata conference for achieving a level of health for the people of the world as the cost of secondary and tertiary care rose beyond the reach of the public. The rise in the cost of health care services has made it difficult for people to access care especially, the poor in rural areas of developing countries who, resort to alternative medicine, as their only option. It was this concern that led to the Alma- Ata conference, which central them was, "bringing health care to the doorsteps of the people living in rural areas" and at a cost they can afford.

Primary health care (PHC) is defined as "essential health care based on practical scientifically sound and socially acceptable methods and technology made universally accessible to individuals and families in the community through their full participation and at a cost that the community and country can afford to maintain at every stage of their development in the spirit of self reliance and self determination" (Alma- Ata declaration, 1978).

The components of PHC, according to World Health Organisation (WHO, 1978), were given as health education, nutrition and food supply, water and sanitation, immunization, maternal and child health services including family planning (M.C.H/F.P), prevention and control of locally endemic diseases, treatment of common ailments and injuries, provision of essential drugs and supply (Alma- Ata conference, 1978). However, two more components have been added, namely; provision of mental health and dental health services (WHO, 1978).

Maternal health which is one of the components of PHC, means ensuring that all pregnant women receive the care they need to be safe and healthy throughout pregnancy and child birth. WHO, (2018) defined maternal health as the health of women during pregnancy, child birth and the post partum period, it compasses the health care dimensions of family planning, preconception, prenatal and post natal care in order to reduce maternal morbidity and mortality. Preconception care can include education, health promotion, screening and other interventions among women of productive age to reduce risk factors that might affect future pregnancies. Following the importance of PHC, the Nigerian government has provided primary health care facilities in all local government areas in order to ensure that pregnant women receive the needed healthcare, but these facilities are not fully utilized. To support this fact, Abdulraheem, Olapipo and Amodu (2012), documented that PHC facilities are relatively and uniformly distributed throughout local government areas in Nigeria, but the rural population do not make adequate use of these facilities. Anderson and Newman (1997) revealed that utilization of health care facilities is a complex behavioural phenomenon, especially among pregnant women in rural areas 
who are so inclined to their traditions and health beliefs.

Apart from traditional and health beliefs, the poor utilization of health services in developing nations especially Nigeria is further heightened by high cost of services, poor or negative attitudes of health staff, poor facilities, ignorance of the importance of utilizing health services especially as it relates to postnatal care mothers, have all been identified as some of the barriers to health care utilization and adequate utilization of health facilities. This is in line with the study of Dhaher, Mikolajczyk, Maxwell \& Krämer, (2008) that revealed that, the most frequent reason for not obtaining postnatal care was that women did not feel sick and therefore did not need postnatal care $(85 \%)$, followed by not having been told by their doctor to come back for postnatal care (15.5\%). Similarly, the study of Titaley, Hunter, Heywood and Dibley (2010) revealed that financial difficulty emerged as the major issue among women who did not fulfill the minimum requirements of four antenatal care services or two postnatal care services within the first month after delivery. Other factors that affected the subjects utilization of their health services includes cost of health services, transportation costs, or both, distances from health facilities, poor road conditions were major concerns, particularly for those living in remote areas, lack of community awareness about the importance of health services, as some community members perceived health services to be necessary only if obstetric complications occurred. Also the utilization of the services of traditional birth attendants for antenatal, delivery, and postnatal care of the women was also one of the problems that impeded the utilization of their health facilities

\section{Statement of the Problem}

The importance of PHC utilization cannot be over emphasized, this is why Oliatan and Mbah, (1985) documented that, regular antenatal visits to health facilities by pregnant mothers is important as all complaints about her and the fetus are examined and cared for. In the process, series of test are conducted for proper diagnoses and treatment of both mother and child. Unfortunately many mothers do not utilize health facilities adequately, even among the few that manage to attend antenatal services majority do not go for postnatal care (Dhaher, Mikolajczyk, Maxwell \& Krämer , 2008)

. These practices are not only endangering the health of the mothers alone, as their babies are even more at risk of internal health problems that may not be known to the parents. These practices have severally resulted to both maternal and child morbidity and mortally. In an attempt to reduce the incidence and prevalence of maternal and child morbidity and mortality, the Government of Nigeria provided and distributed PHC facilities throughout all the local government areas with the belief that inadequate health facilities were the root cause of these poor utilization which culminate into pregnancy related morbidity and mortality.

Despite these efforts in providing and distributing PHC facilities throughout local government areas in Nigeria, the rural populations do not adequately utilize the available basic health care facilities. This is very worrisome and pathetic. Therefore, it is not known to the researchers whether pregnant women in Ikpoba - Okha equally fall into this category of people that do not adequately utilize the available basic health care facilities, because there is no empirical work done in this area that is known to the researchers. It was against this back ground that this study was carried out to find out the factors that impede adequate utilization of PHC facilities by Ikpoba- Okha pregnant women, with particular interest in the socio-cultural, socio-economic, government and health system factors as well as poor infrastructure, equipment, accessibility, quality health care, shortage of health staff, cost of services and attitude of health care provider.

\section{Research Questions}

The following research questions were raised to guide the study

1. Do cultural beliefs constitute an impediment to adequate utilization of primary health care facility by pregnant women?

2. Does the cost of PHC services affect their utilization?

3. Do pregnant women have problems with distance to available health care facilities?

4. Does attitude of health staff influence health care utilization among pregnant women?

5. Does government nonchalant influence affect adequate PHC utilization?

6. Does shortage of health personnel constitute an impediment to adequate PHC utilization?

\section{Purpose of the Study}

The purpose of this study was to identify the factors that impede adequate utilization of primary health care facilities by pregnant women in Ikpoba -Okha Local Government Area of Edo state. The impact of the following variable: socio- cultural, socio -economic, health system and governmental factors were also examined. To proffer intervention strategies from the result of the study, for improving the current low utilization of health care facilities by pregnant women. 


\section{Research Design}

The descriptive survey design was adopted in the study because authorities in research methodology such as Owie, (2006) and Nwana (1990) have maintained that it provides appropriate methodology for investigating human behaviour and perception when population under study is large, it is considered necessary as the data collected from the study sample serve as representative of the entire population.

\section{Population of the Study}

The population of study consists of all pregnant mothers numbering two thousand four hundred and ninety seven (2497), who access and utilize primary health care facilities for maternal health care in rural communities of Ikpoba Okha Local Government Area of Edo State as at the time of research. The fatalities are: Amufi health centre, Avbiama health care centre, Avbagba health centre, Idogbo health centre, Iyanomo health centre, iyenugho health centre, ikpe health centre, Obayantor health centre, Okhabere health centre, Oghoghobi health centre, Obagie health centre, Ohovbe health centre, Egba community health centre, Umelu health centre, Ukhiri Health centre, Uteh health centre and Ugbekun health centre. (Source Statistic Unit, Idogbo, 2012).

\section{Sample and Sampling Technique}

The sample size for the study was 270 respondents and it was selected through the multi stage sampling procedures. In the first stage, the facilities were serialized into 1-18, thereafter, a systematic random sampling technique was applied to select (9) nine of the health facilities, this was done by choosing all odd numbers, while the purposive sampling technique was adopted to select 30 pregnant women each from the nine facilities to get a total of 270

\section{Research Instrument}

Two research instruments were used in the study, a checklist and a 35 item self structured questionnaire titled "factors affecting adequate utilization of PHC facilities" in rural areas. The questionnaire consists of two sections (section A and B). Section A deals with educational level, occupation, residence while Section "B" elicits responses on the factors that impede utilization of primary health care facilities. Illiterates were accessed through translating the items in the questionnaire into their local language.

\section{Validity of the Instrument}

The instrument was content validated by three experts in health education. Their recommendations and corrections were used in the final draft of the instrument.

\section{Reliability of the Instrument}

The test-retest method was used to ascertain the reliability of the instrument. This involved first, administering the instrument to some selected pregnant mothers from the same clusters that will not take part in the study and after a period of about two weeks, same instrument was administered to same population, the scores obtained from both administrations were computed using the Pearson's product moment correlation coefficient.

\section{Administration of the Instrument}

A letter of permission to distribute the instrument stating the purpose of the research was submitted to the Head Office at Idogbo by the researchers and was endorsed by the management to the heads of primary health centres in Idogbo. The instruments were administered by the researchers with the aid of trained two research assistants by the researcher. The instruments were also collected as soon as the respondents finished responding to them.

\section{Data Analysis}

The data obtained were analyzed using frequency counts, mean scores and percentages.

Out of the 270 questionnaires administered only 250 were found useful and this constituted the basis for the analysis. Some were mutilated and some were not returned.

Data collected were analyzed discussed in line with the research questions.

\section{Table1: Cultural Belief as an Impediment to PHC Utilization}

\begin{tabular}{|l|l|l|l|}
\hline S/N & Items & Yes & No \\
\hline 1. & Do your cultural belief influence your choice of health care utilization & $150(60)$ & $100(40)$ \\
\hline 2. & Does your husband decide where you get health care? & $180(80)$ & $70(20)$ \\
\hline 3. & Do you prefer the service of TBAs for your maternal care? & $140(56)$ & $110(44)$ \\
\hline 4. & $\begin{array}{l}\text { My culture does not permit my acceptance of blood transfusion when the } \\
\text { need arises? }\end{array}$ & $210(84)$ & $40(16)$ \\
\hline 5. & Does your culture influence your choice & $140(56)$ & $110(44)$ \\
\hline & Mean or average score & $(66.4)$ & \\
\hline
\end{tabular}


Table1 showed the influence of cultural belief on adequate utilization of PHC facilities by pregnant women in Ikpoba Okha. The table1 revealed that: $60 \%$ of the women maintained that cultural beliefs influence their choice of PHC utilization, 56\% maintained that, they prefer the services of TBAs for maternal mentality to orthodox, 84\% reported that their culture does not allow them to accept blood transfusion if the need arises, while $56 \%$ reported that their culture influence the choice of their health care. The table1 above also showed that the mean score of cultural belief constituting an impediment to adequate utilization of PHC is $66.4 \%$. Since the average score to research question 1 is more than $50 \%$, it therefore means that cultural belief constitutes an impediment to adequate utilization of PHC facilities among pregnant women in Ikpoba-Okha LGA of Edo State.

Table 2: Influence of Cost on PHC Services Utilization

\begin{tabular}{|l|l|l|l|}
\hline S/N & Items & Yes & No \\
\hline 6. & Is cost of antenatal registration too much? & $220(88)$ & $30(12)$ \\
\hline 7. & Are the drugs given to you expensive & $130(52)$ & $120(48)$ \\
\hline 8. & Do you pay any form of tax in the health care fertility? & $50(20)$ & $200(80)$ \\
\hline 9. & Do you pay for clinic card when mistakenly lost? & $150(60)$ & $100(40)$ \\
\hline & & $(55)$ & \\
\hline
\end{tabular}

Table 2, revealed the influence of cost of health services to adequate utilization of PHC among pregnant women in Ikpoba-Okha LGA of Edo State. The table 2 above revealed that: $88 \%$ of the women responded that the cost of antenatal registration is too expensive for them to afford, while $52 \%$ maintained that the drugs given to them in PHC are also too expensive for them to afford, and lastly, $60 \%$ of the women reported that, they are always asked to pay for their cards if mistakenly lost. Again, the table 2 above revealed that more than half $(55 \%)$ of the women reported that cost of PHC services is an impending factor to their adequate utilization of PHC services.

Table 3: Influence of Distance to PHC on Utilization

\begin{tabular}{|l|l|l|l|}
\hline S/N & Items & Yes & No \\
\hline 10. & Do you trek a long way before getting to the health care centre? & $210(84)$ & $40(16)$ \\
\hline 11. & Does distance discourage you from using facility? & $215(86)$ & $35(14)$ \\
\hline 12. & There is no available taxi cab to the health centre. & $125(90)$ & $25(10)$ \\
\hline 13. & Is PHC centre far from your residence? & $200(80)$ & $50(20)$ \\
\hline 14. & I do not have a private means of transport to health centre. & $235(94)$ & $15(06)$ \\
\hline & & $(86.8)$ & $(13.4)$ \\
\hline
\end{tabular}

Table 3, revealed the influence of distance to PHC on adequate utilization of PHC facilities by pregnant women in Ikpoba-Okha. The table 3 above revealed that: $84 \%$ of the respondents do trek a long distance before getting to their health care centres, $86 \%$ are discouraged from using PHC because of distance from their house, the non availability of taxi or cab to PHC force $90 \%$ of the respondents to trek to their various health centres, $80 \%$ complained that the PHC are cited too far from their places of residence, while $94 \%$ maintained that they do not have a private means of transport to health centres. The table4 also revealed that majority $(86.8 \%)$ of the respondents attested to the fact that distance affects their adequate utilization of PHC facilities.

Table 4: Attitudes of Health Staff as an Impediment to PHC Utilization

\begin{tabular}{|l|l|l|l|}
\hline S/N & ITEMS & YES & NO \\
\hline 15. & $\begin{array}{l}\text { Do health care providers shout at you in the course of providing health care } \\
\text { services? }\end{array}$ & $128(51)$ & $122(49)$ \\
\hline 16. & I do not relate freely with health care providers? & $135(54)$ & $15(46)$ \\
\hline 17. & Do health care providers come late to work? & $123(49)$ & $127(51)$ \\
\hline 18. & Do care providers show commitment to work & $123(49)$ & $127(51)$ \\
\hline 19. & Do you think some of the care givers are wicked? & $140(56)$ & $110(44)$ \\
\hline & \multicolumn{2}{|l}{$(51.8)$} & \\
\hline
\end{tabular}

Table 4, revealed the influence of attitudes of health staff to adequate utilization of PHC among pregnant women in Ikpoba-Okha. The table4 above revealed that: $51.8 \%$ of the respondents complained shout at them while providing with health care services, $54 \%$ maintained that they do not relate freely with their health care providers because of their poor attitudes towards them, while 56\% reported that some of their health care providers are very wicked. The table 4 above also revealed that more than half $(51 \%)$ of the respondents maintained that attitudes of health staff impede them their adequate utilization of PHC facilities in their areas. 
Tables 5: Government Related Issues as an Impediment to PHC Utilization

\begin{tabular}{|l|l|l|l|}
\hline S/N & ITERMS & YES & NO \\
\hline 20. & Does poor infrastructure affect utilization? & $200(80)$ & $100(20)$ \\
\hline 21. & The referral system is not functional. & $240(93)$ & $20(07)$ \\
\hline 22. & The equipment in our PHC are not in good working condition. & $230(92)$ & $20(08)$ \\
\hline 23. & Are routine drugs readily available & $135(54)$ & $115(46)$ \\
\hline 24. & Are vaccines for pregnant women and infants always available? & $120(48)$ & $130(52)$ \\
\hline & & $(73.4)$ & \\
\hline
\end{tabular}

Table5 showed the analysis of the influence of government nonchalant attitudes has over adequate utilization of PHC by pregnant women in Ikpoba-Okha LGA of Edo State. Table 5 also revealed that; poor infrastructure in PHC affected $90 \%$ of the respondents from utilizing PHC adequately, the non functionality of the referral system also impede $93 \%$ of the respondents from using PHC facilities adequately, while $92 \%$ of the respondents maintained that the poor working conditions of the equipment in their PHC constitute an impediment to adequate utilization of PHC. Lastly, table 5 showed that total mean score of the respondents on whether government nonchalant attitude influence their adequate utilization of PHC was $71.8 \%$. In other words, $71.8 \%$ of the respondents maintained that government nonchalant attitude influence their adequate utilization of PHC.

Table 6: Shortage of Health Personnel as an Impediment to PHC Utilization

\begin{tabular}{|l|l|l|l|}
\hline S/N & ITEMS & YES & NO \\
\hline 25. & Do you wait for a long time before you are attended to? & $128(51)$ & $122(49)$ \\
\hline 26. & $\begin{array}{l}\text { Using the health facility can be very stressful for both the health staff } \\
\text { and their patients }\end{array}$ & $130(52)$ & $120(48)$ \\
\hline 27. & Are the health staff motivated? & $130(52)$ & $120(48)$ \\
\hline & & $(51.3)$ & \\
\hline
\end{tabular}

Table 6 showed the influence of shortage of health staff over adequate utilization of PHC by pregnant women in Ikpoba okha. The table6 above also showed that more than half $(51 \%)$ of the women maintained that they do wait for a long time before they are attained to, 52\% reported that they do not utilize PHC adequately because using health facilities in their area can be very stressful for both the health staff and patient especially, while 52\% reported that from their observation, health staff are not motivated to work. Table6 above further showed that more than half $(51.7 \%)$ of the respondents agreed that shortage of health staff constitute an impediment to their adequate utilization of PHC.

\section{Discussion of Results}

This discussion of results is done according to the research questions as follow:

Research question 1 revealed that cultural beliefs constitute an impediment to adequate PHC utilization; the percentage score of the respondents show more than $50 \%$ of responses attesting to the fact that cultural beliefs affect utilization. The findings of this study agrees with that of Ajiboye and Adebayo (2012) where positive significant relationship was found between socio-cultural factors and pregnancy outcome among Ogu speaking people of Badagry area of Lagos State. The socio-cultural factors ranged from economic status of pregnant women and their spouses, number of children they have, the year at marriage, level of education, income and reactions to cultural practices during pregnancy.

This finding again, agrees with Oke (1996), when he observed that the use and non-use of health services are determined by one's socio-cultural environment, which, in most cases, is shaped by its patriarchal structure. This position was again, corroborated by Erinosho (1998) that many culture bound syndromes are effectively managed through an informed knowledge of their cultural contexts and the background of patients.

Research question 2 revealed that more than $50 \%$ of respondents indicated that cost of health service is an impending factor to adequate utilization of PHC facilities. This collaborates the findings of National Population Commission of Nigeria and ICF (NDHS, 2014) that $8 \%$ of women did not deliver in a health facility because of unaffordable cost and as many as $56 \%$ of women could not afford the cost of antenatal care.Again, to support this, Saha and Ravindran (2002) asserted that increased cost of medicines and payments for services hinder accessibility to health care centers'. In the same vein, Ako, Oloyede, Okonlawon and Odusoge (2006), noted that cost of health services and quality of the care were found to be contributory factors to inadequate utilization of PHC facilities. On a contrary, the study of Anders (1985) observed that among the sociodemographic variables neither level of education nor income had any influence on utilization when other variables were taken into account.

Research quetion3 revealed that distance to primary health care centre impede adequate utiliztion of same, this is evident in the responses of the respondents in all the items of the questionnaire, more than $50 \%$ indicated that distance affect utilization of FHC facilities. This agrees with the result from the study of Saad, (2004) that a 
number of patient-related variables (income, health status, education, gender, the presence of health insurance and nationality) and provider-related variables (location of the health facility, waiting time, the availability of ancillary services, opening hours and the availability of specialised doctors) were statistically significant in identifying the variables which influence the utilisation of both public PHC centres and private outpatient clinics. This again corroborate with the finds from the study of Al-Doghaither, et al (2003) were the main factors associated with choosing a hospital were medical services, accessibility, age, sex and education.

Again, the findings of this study support those of Panezai, Ahmad and Saqib (2017) that the convenience of the Basic Health Unit location had a significant positive influence on access to PHC services of their women. The findings of this study further corroborate those of Mateen, Shaikh, and Kumar (2013), who showed the importance of having suitable locations for health facilities because of the limited availability of transport facilities, the long distances between scattered villages, and the uneven landscape.

Research question 4, revealed that attitude of health staff impedes adequate utilization of health care services, this can be seen from the responses where $51 \%$ of the respondents indicated that health providers shout at them in the course of providing care, $54 \%$ of the respondents say they do not relate freely with care providers, $56 \%$ of the respondents say some of the care providers are wicked, $51 \%$ of the respondents say that care providers show commitment to work. This agrees with the findings of AlOmar and Saeed (1999) were perceived quality of medical staff of which attitude is not left out among other variables such as source of payment, availability of other sources of income, distance between residence and primary health care provider, education, preference for a doctor of the same gender, preference for a Saudi doctor were the most influential factors in discriminating between the users of public and private health care facilities. In the same vein, it agrees with Saeed (1998) that the choice of hospital is influenced by socio-demographic variables as well as hospital attributes. It should be noted here that the attitudes of health staff is one of the factors that constitutes these hospital attributes.

Research question 5 revealed that government nonchalant attitudes impede adequate utilization of PHC facilities. This is evident in responses of the respondents which have shown more than $50 \%$ yes in all the items of the questionnaire on government nonchalant attitude. This agrees with Gupta (2004) that lack of maintenance culture of the health facilities, infrastructural decay, lack of equipment, non-referral system, lack of basic emergency obstetric care and lack of monitoring and supervision reduce the faith of the intending user. Similarly, Wunsch and Olowu (1996), found that lack of maintenance culture of the health facilities, most vehicles donated by UNICEF in the 1980s are totally non-functional making it difficult for health workers to perform their tasks especially for immunization out reach.

Research question 6, revealed that shortage of health personnel affect utilization, this is evident in the responses of the respondents with more than $50 \%$ agreeing that they wait a long time before they are attended to, using the facility can be very stressful for both the health personnel and the pregnant women. This equally is in line with the finding of Saad, (2004) that waiting time, opening hours and the availability of specialised doctors among other factors were statistically significant in identifying the variables which influence the utilisation of both public PHC centres and private outpatient clinics.

Based on the findings of the study, the following were deduced:

1. Socio-cultural beliefs impede adequate utilization of PHC facilities

2. Attitudes of health staff affect PHC utilization in Ikpoba-Okha

3. Shortage of health personnel has been found to impede adequate utilization of health care services.

4. Cost of services also discourages pregnant women from utilizing PHC services and government related issues. Government should provide adequate supervision, monitoring and evaluation of programmes and should be pursued with vigor and required manpower.

\section{Recommendations}

Primary Health Care utilization is essential in actualizing Millennium Development Goals 5 (MDGs) reducing maternal mortality by half by the year 2015. Hence, the following recommendations may go a long way in the quest for a lasting solution in the campaign to accelerate the utilization of health facilities in Ikpoba-Okha LGA Edo State, Nigeria and other regions of the worlds:

1. Health education be intensified in creating public awareness on the importance of using PHC facilities among pregnant women and that harmful traditions should be discarded.

2. Costs alleviation for women seeking antenatal care and delivery services should be put in place to encourage women to patronize health services.

3. Restoration of the dignity of women through education and empowerment for the prospective mothers. Generally, the use of maternal health care services increases positively with education. Both education and good occupation will bring women on board the decision-making realm including health care. In addition, education may increase knowledge which will reduce early marriage and pregnancies among women in the study area.

4. Efforts should be made to train the traditional birth attendants to refine their operations and lessen the havoc 
caused by them. As we know, the use of modern health services is often influenced by individual perception or feelings of the efficacy of the services.

5. Orientation should be conducted for health workers on how to uphold the ethics and on rudimentary principles of human relations to make better friendly service.

6. Maternal health care services increase positively with education, community members should be carried along in planning and in decision-making processes on health care matters.

\section{REFERENCES}

Ajiboye, O.E., Adebayo, K.A., 2012. Socio-Cultural Factors Affecting Pregnancy Outcome Among the Ogu Speaking People of Badagry Area of Lagos State, Nigeria. International Journal of Humanities and Social Science 2, 133-144.

Al-Doghaither, A., Abdelrhman, B., Saeed, A. and Magzoub, M.E. (2003) Factors Influencing Patient Choice of Hospitals in Riyadh, Saudi Arabia, The Journal of The Royal Society for the Promotion of Health, 123(2): 105-109.

Al-Omar, B. and Saeed, K. (1999) Factors influencing patient's utilization of primary health care providers in Saudi Arabia, Journal of Family and Community Medicine, 6(1) 31-39.

Anders, G. H. S. (1985). Factors Affecting Primary Health Care Utilization. Family Practice 1, (3), $155-161$. https://academic.oup.com/fampra/article- abstract/1/3/155/775768?redirectedFrom=PDF

Bakeera, S. K, Wamala, S.P, Galea, S. State, A, Peterson, S., and Pariyo, G.W (2009). Community perceptions and factors influencing utilization of health services in Uganda. International journal for equity in health

Dhaher, E, Mikolajczyk, R.T, Maxwell, A.E \& Krämer, A. 2008). Factors associated with lack of postnatal care among Palestinian women: A cross-sectional study of three clinics in the West Bank. BMC Pregnancy and Childbirth. Retrieved from https://bmcpregnancychildbirth.biomedcentral.com/articles/10.1186/1471-239310-61

Erinosho, O. A. (1998) Health Sociology, Ibadan: Sam Bookman.

Gupta, M.D., Guari, V. \& Khemani, S. (2004). Decentralized delivery of primary health services in Nigeria

National Population Commission of Nigeria \& ICF Macro (2014). Nigeria 2013 Demographic and Health Survey, Abuja, Nigeria. $\quad$ Retrieved from https://www.researchgate.net/publication/303752425_Maternal_Health_in_Nigeria_A_sit uation_update

Nwana, O.C. (1990). Introduction to education research for student teachers. Ibadan: Heinemann Books Ltd.

Oke, E. A. (1996) The Emergence of Medical Sociology; In E. A. Oke and B. E. Owumi (eds) Readings in Medical Sociology. Ibadan: Resource Development and Management Services, PP. $1-14$.

Oke, E. A. (1996) The Emergence of Medical Sociology; In E. A. Oke and B. E. Owumi (eds) Readings in Medical Sociology. Ibadan: Resource Development and Management Services, PP. 1 - 14

Owie, I. (2006). Fundamentals of statistics in education and social sciences. Benin City, Mindex.

Saad, A. A.(2004). Factors Influencing the Utilisation of Public and Private Primary Health Care Services in Riyadh City. JKAU: Econ. \& Adm., 19, (1), 3-27. )

Saeed, K. (1998) Factors affecting patients' choice of hospitals, Annals of Saudi Medicine, 18(5): 420-424.

Titaley, C.R., Hunter, C.L., Heywood, P. \& Dibley, M.J. (2010). Why don't some women attend antenatal and postnatal care services?: a qualitative study of community members' perspectives in Garut, Sukabumi and Ciamis districts of West Java Province, Indonesia. BMC Pregnancy and Childbirth. Retrieved from https://bmcpregnancychildbirth.biomedcentral.com/articles/10.1186/1471-2393-10-61

World Health Organization (1978). Ama-Atta, report of International conference of Primary Health Care. https://apps.who.int/iris/handle/10665/39228

World Health Organization, (2018). Maternal health. Retrieved from https://en.wikipedia.org/wiki/Maternal_health 